PAPER

\title{
Apolipoprotein E genotypes do not influence the age of onset in Huntington's disease
}

\author{
C Saft, J E Andrich, N Brune, M Gencik, P H Kraus, H Przuntek, J T Epplen
}

See end of article for authors' affiliations

.....................

Correspondence to: DrJ E Andrich,

Department of Neurology, St Josef Hospital, RuhrUniversity Bochum,

Gudrunstr. 56, D-44791

Bochum, Germany;

jürgen.andrich@

ruhr-uni.de

Received 6 July 2003

In revised form

25 January 2004

Accepted

18 February 2004
J Neurol Neurosurg Psychiatry 2004;75:1692-1696. doi: 10.1136/jnnp.2003.022756

Objective: The $\epsilon 4$ allele of the apolipoprotein $E$ (ApoE) gene has been defined as a critical factor for early onset neurodegeneration in Pick's, Parkinson's, and Alzheimer's disease. Unexpectedly, the $\epsilon 4$ allele appeared to delay the age of onset in Huntington's disease (HD) patients. Furthermore, sex specific effects were reported on earlier age of onset due to the $A p o E \in 2 \in 3$ genotype in males with $H D$. The age of onset of $H D$ is known to be negatively correlated with increasing lengths of pathogenetic CAG expansions in the huntingtin gene.

Methods: In order to examine the effects of CAG block lengths, we have correlated ApoE genotypes with the age of onset in 145 patients symptomatic for HD with psychiatric and somatic symptoms (depression, psychosis, dementia, choreic, and other movement disorders) harbouring only modestly expanded huntingtin alleles (41-45 CAGs).

Results: The negative correlation between age of onset and CAG block length was established in our HD cohort. Statistically significant effects of the $\epsilon 4$ allele were not obvious regarding clinical characteristics including age of onset, nor were any sex differences for the $€ 2 € 3$ genotype observed.

Conclusion: The ApoE genotype does not affect the course of HD significantly.
$\mathrm{H}$ untington's disease (HD) is an autosomal dominantly transmitted neurodegenerative disorder based on expansions of translated CAG repeats in the huntingtin gene beyond a threshold of 36 to $>200$ units. $^{1}$ Downstream effects of the mutation involve disturbances of gene transcription and neurotransmission, protein misfolding, and accumulation as well as apoptosis. ${ }^{2}$ The age of onset in HD is negatively correlated with the CAG repeat length. ${ }^{3}$ This correlation, however, only explains approximately $50-70 \%$ of the variation in the age of onset. ${ }^{4}$ Particularly, other genetic and environmental variables are assumed in cases with limited CAG expansions. ${ }^{6}$ As in other neurodegenerative diseases, different alleles of the apolipoprotein E (ApoE) gene are candidates for influencing the age of onset in HD.

The ApoE protein is a component of very low density lipoprotein (VLDL) particles and chylomicrons and is involved in lipid transport. ${ }^{7}$ The pathophysiological effects of the $\epsilon 4$ allele in neurodegeneration may involve reduced neuroprotection against amyloid depositions, reactive oxygen species, and excitotoxins. ${ }^{8}$ There are three common protein isoforms of ApoE: $\epsilon 2, \epsilon 3$, and $\epsilon 4$. The $\epsilon 4$ allele has been identified as a risk factor in Alzheimer's (AD) ${ }^{9}{ }^{10}$ and Pick's disease. ${ }^{11}$ Furthermore, increased susceptibility to sporadic Parkinson's disease by a certain combined $\alpha$-synuclein/ApoE genotype has been identified. ${ }^{12}$ In contrast to the aforementioned findings, the $\epsilon 4$ allele has been described to delay age of onset in patients with HD. ${ }^{13}$ Furthermore, gender specific effects were observed, that is, an earlier age of onset due to the ApoE $\epsilon 2 \epsilon 3$ genotype in males with HD. ${ }^{14}$ In both studies, designed to detect the influence of genetic variables other than CAG repeats, cohorts with broad repeat ranges were investigated (40-57 CAG units, $\mathrm{n}=60^{13} ; 38-67$ CAG units, $\left.\mathrm{n}=138^{14}\right)$. On the other hand, similar effects of ApoE genotypes were not demonstrable in independent investigations covering broad ranges of CAG expansions. ${ }^{511}$ In order to minimise the influence of the CAG repeat length, we have studied patients with a very narrow range of CAG repeats (41-45) in a homogeneously characterised HD cohort from the Huntington Center NRW, Germany.

\section{METHODS}

Clinical assessment of 420 patients with HD was performed by two experienced members of the Huntington Center, blinded with respect to DNA test results. Initially, 167 mutation carriers with 41-45 CAG units were selected from this group. Clinical assessment had been performed using a standardised battery of neurological (motor and behavioural score according to the Unified Huntington's Disease Rating Scale (UHDRS), apparative testing of motor function such as peg insertion and tapping), ${ }^{15}$ neuropsychological (Beck Depression Inventory (BDI), Benton-Test, Wechsler Memory Scale, subtests from the Aachen-Aphasia-Test, Clock-Test), and radiological investigations (cranial computer tomography atrophy markers) in order to determine symptoms and (motor and/or psychiatric) age of onset. Patients with an UHDRS score of more than 5 were included as symptomatic for HD. Symptoms were categorised as choreic movements, other movement disturbances, dementia (mild cognitive impairment to severe dementia according to neuropsychological testing), depression, and psychosis (according to ICD-10 classification). One predominant symptom was defined according to the patient's/caregiver's statement or investigator's impression. All patients had given informed consent for molecular genetic analysis. This study has been approved by the ethics committee of the Ruhr-University (\#1457/2000).

CAG repeat lengths were determined after PCR amplification of genomic DNA from peripheral white blood cells. CAG repeats were amplified by established methods. ${ }^{16}{ }^{17}$

For ApoE restriction isotyping, flanking primers ApoE F (5'-CGGGCACGGCTGTCCAAGGA-3') and ApoE R (5'GGGCCCCGGCCTGGTACAC-3') were used to amplify part of the ApoE gene containing amino acid positions 112 and 158. PCR reactions were performed in a volume of $10 \mu \mathrm{l}$ containing $200 \mathrm{ng}$ DNA, 4 pmol of each oligonucleotide, $0.2 \mathrm{mmol}$ each of dATP, dGTP, dTTP, and dCTP, $1 \mu \mathrm{l}$ of $\left[\gamma_{-}{ }^{32} \mathrm{P}\right] \propto \mathrm{dCTP} \quad(3000 \mathrm{mCi} / \mathrm{mmol}), 3 \mathrm{mM} \quad \mathrm{MgCl}_{2}, \quad 0.5 \mu \mathrm{l}$

Abbreviations: $A D$, Alzheimer's disease; ApoE, apolipoprotein $E$; $H D$, Huntington's disease; UHDRS, Unified Huntington's Disease Rating Scale 
Table 1 Predominant symptoms in 151 HD patients according to length of CAG block

\begin{tabular}{|c|c|c|c|c|c|}
\hline \multirow[b]{2}{*}{ Symptoms } & \multicolumn{5}{|c|}{ CAG block length } \\
\hline & $41(n=20)$ & $42(n=38)$ & $43(n=43)$ & $44(n=35)$ & $45(n=15)$ \\
\hline Choreic movements & $11(55.0 \%)$ & $15(39.5 \%)$ & $22(51.1 \%)$ & $18(51.4 \%)$ & $11(73.3 \%)$ \\
\hline Depression & $3(15.0 \%)$ & $7(18.4 \%)$ & $12(27.9 \%)$ & $7(20.0 \%)$ & $2(13.3 \%)$ \\
\hline Dementia & $5(25.0 \%)$ & $11(28.9 \%)$ & $3(7.0 \%)$ & $7(20.0 \%)$ & $2(13.3 \%)$ \\
\hline Psychosis & $0(0 \%)$ & $3(7.9 \%)$ & $6(14.0 \%)$ & $2(5.7 \%)$ & $0(0 \%)$ \\
\hline Other movement disorders & $1(5.0 \%)$ & $2(5.3 \%)$ & $0(0 \%)$ & $1(2.9 \%)$ & $0(0 \%)$ \\
\hline
\end{tabular}

formamide, $1 \mathrm{U}$ Taq polymerase, and $1 \mu \mathrm{l}$ of PCR buffer without $\mathrm{MgCl}_{2}$. The reaction mixture was subjected to 30 cycles of amplification by primer annealing $\left(61^{\circ} \mathrm{C}\right.$ for $\left.1 \mathrm{~min}\right)$, extension $\left(72^{\circ} \mathrm{C}\right.$ for $\left.1 \mathrm{~min}\right)$, and denaturation $\left(94^{\circ} \mathrm{C}\right.$ for $1 \mathrm{~min}$ ). Then, $2.5 \mathrm{U}$ of Hin61 (MBI Fermentas, St Leon-Rot, Germany) were added in a volume of $20 \mu \mathrm{l}$ containing $3 \mu \mathrm{l}$ of $10 \times$ Yellow buffer $(\mathrm{MBI})$ and $3 \mu \mathrm{l}$ of $10 \times$ bovine serum albumin to each reaction mixture. After incubation for $3 \mathrm{~h}$ at $37^{\circ} \mathrm{C}$, restriction fragments were separated on $8 \%$ non-denaturing polyacrylamide gels and visualised by autoradiography.

Statistical analyses included $t$ tests and $\chi^{2}$ and nonparametric tests (Mann-Whitney). Spearman's $\rho$ was used for correlation analysis. In order to exclude any possible interference by the number of CAG repeats on our analyses, we also performed multivariate regression analysis for an exponential model. The influence of parameters that otherwise could possibly cause bias (for example, age, sex) was excluded by conducting separate analyses (correlation and discriminant analyses).

\section{RESULTS}

HD symptoms in individuals carrying expanded CAG repeat blocks

Of our DNA test cohort (carrying 41-45 CAG repeats) 13 presymptomatic counselees had asked for predictive testing and were excluded from further investigations. A total of 154 patients showed symptoms of HD. Two pairs of these patients were related and, therefore, one of each pair was excluded randomly. Another excluded patient with two expanded HD alleles (43 and 37 CAGs) did not show additional clinical phenomena compared to those with the commonly observed expansion in one single huntingtin allele. Nearly all the remaining 151 (71 female, 80 male) patients (97.4\%) shared symptoms of chorea, with $51.0 \%$ suffering from choreic movements as the predominant clinical feature. Of the patients $29.8 \%$ (2.6\% as lead symptom) presented other movement disturbances such as dystonia, tremor, myoclonus, or distinct akinesia, while $64.2 \%$ of all patients suffered from symptoms of depression, for example mood and sleep disturbance. Major depression according to ICD-10 was present as the main symptom in $20.5 \%$ of the patients, while $27.8 \%$ suffered from signs of psychotic development with delusions or compulsive behaviour. In $7.3 \%$ severe psychosis with illusions and hallucinations fulfilling ICD-10 criteria was the main symptom. Dementia was found in $83.8 \%$ of all patients (in $18.6 \%$ as the predominant symptom). Concerning CAG block lengths, there were no remarkable differences in the predominant symptoms among the 151 patients. All symptoms such as choreic movements, akinesia, depression, dementia, or psychosis occurred with similar frequencies in the groups with different CAG lengths (table 1).

\section{Age of onset of HD patients}

Mean (SD) age of onset of motor symptoms was 47.51 (8.83) years, while first psychiatric abnormalities were found at the age (SD) of 45.87 (8.97) years. For 30 of the 151 patients it was impossible to evaluate the onset of initial psychiatric abnormalities. In 121 patients with both sets of data for onset of motor symptoms (47.00 (9.12) years) and psychiatric abnormalities (45.76 (9.14) years), there was a significantly earlier onset of psychiatric symptoms $(p=0.001)$ as estimated by the two tailed $t$ test. Using Spearman's $\rho$, there was a significant negative correlation between CAG size and age of motor $(\mathrm{r}=-0.522, \mathrm{p}<0.0005)$ as well as psychiatric onset $(\mathrm{r}=-0.445, \mathrm{p}<0.0005$; fig 1$)$.

\section{Age of onset and sex}

Age of onset (SD) for first motor symptoms in males was $47.04(8.29)$ years $(n=80)$ and in females $48.00(9.50)$ years $(\mathrm{n}=71)$. Initial psychiatric abnormalities in males were
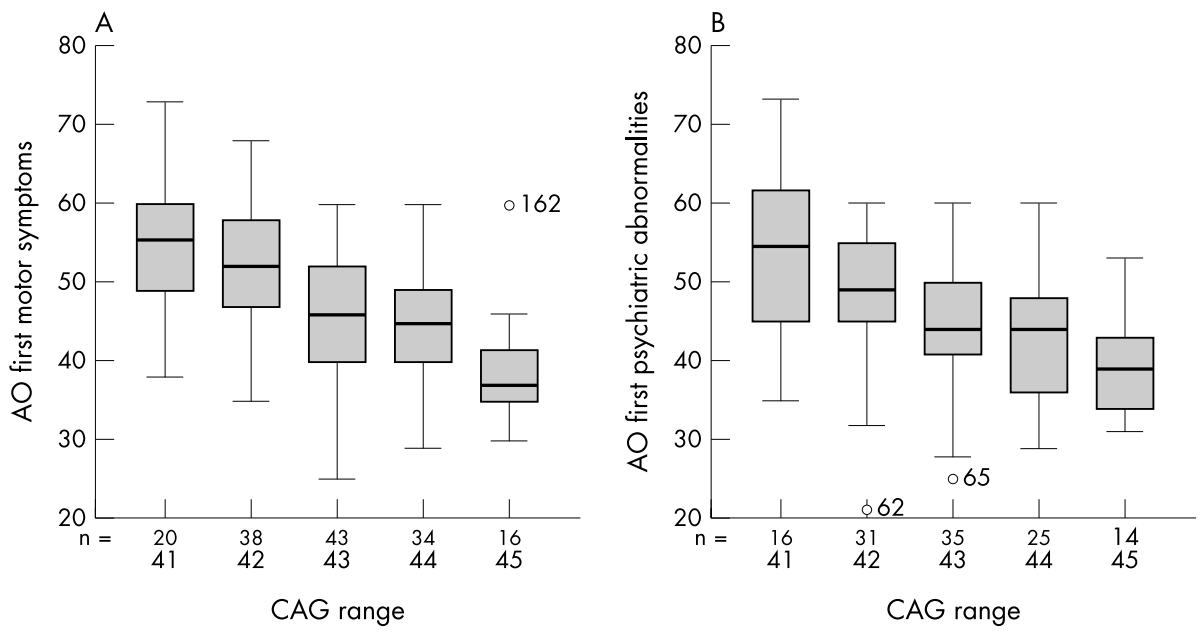

Figure 1 Age of onset (AO) of initial motor symptoms (A) and first psychiatric abnormalities (B) in patients with different CAG block lengths (in years; $n$, number of patients). 

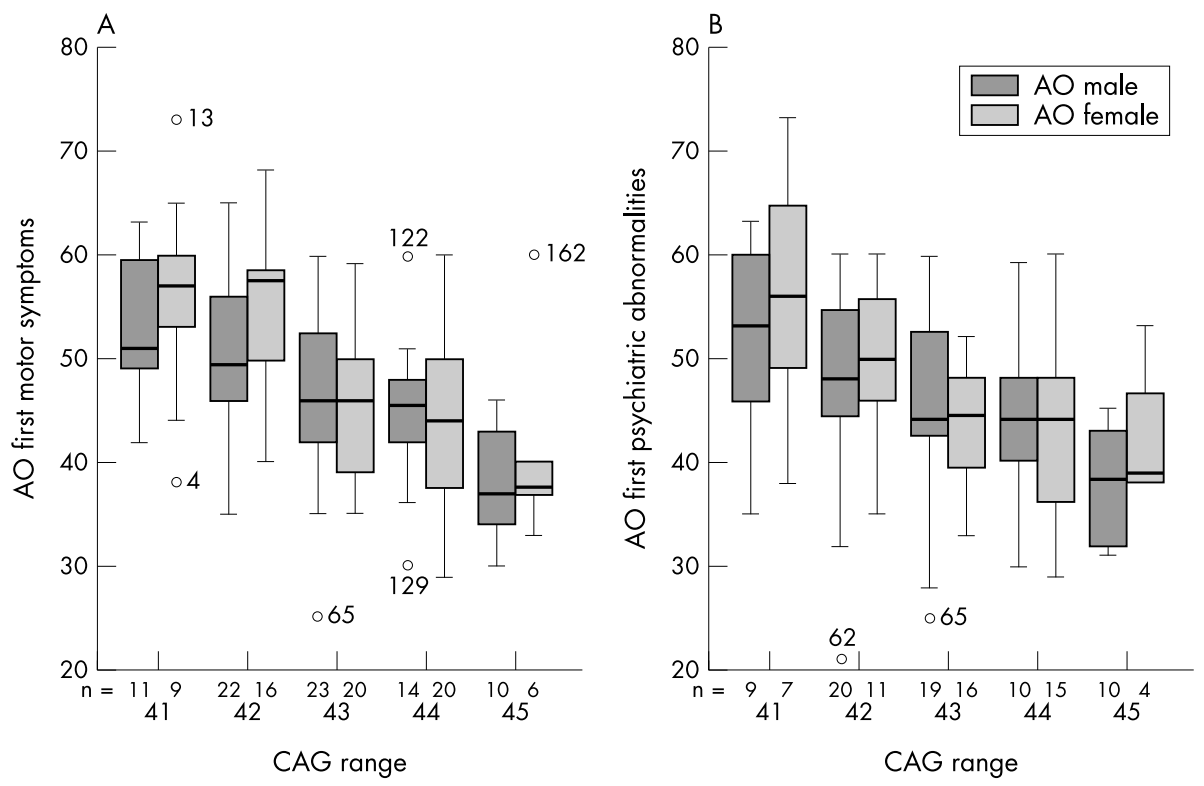

Figure 2 Age of onset $(\mathrm{AO})$ of first motor symptoms $(A)$ and initial psychiatric abnormalities $(B)$ in male and female patients with different CAG block lengths (in years; $n$, number of the patients).

reported at 45.47 (9.17) years of age (no data for 12 patients) and in females at 46.13 (9.17) years of age (data lacking for 18 patients). Spearman's $\rho$ revealed a significant negative correlation between CAG block length and age of onset of first motor symptoms in male $(\mathrm{r}=-0.507, \mathrm{p}<0.0005)$ and female patients $(\mathrm{r}=-0.533, \mathrm{p}<0.0005)$ as well as between CAG range and first psychiatric abnormalities in male $(\mathrm{r}=-0.455, \mathrm{p}<0.0005)$ and female patients $(\mathrm{r}=-0.429$, $\mathrm{p}<0.001$; fig 2). There was no significant difference in age of onset between male and female HD patients in any comparison ( $t$ test). In addition, there were no significant differences regarding CAG repeat lengths between males and females (Mann-Whitney).

\section{Transmission}

In $40 \mathrm{HD}$ cases it was possible to evaluate the age of onset of the patients' parents (mean (SD) age for motor symptoms 48.35 (8.15) years). Focussing on maternal and paternal transmission in these 40 patients, the age of onset of first motor symptoms was 3.96 years earlier in the case of maternal transmission (48.32 (8.41) v 44.36 (6.38) years; $\mathrm{n}=28, \mathrm{p}=0.027)$, but 4.84 years earlier in the case of paternal transmission (48.42 (7.89) $v 43.58$ (7.79) years; $\mathrm{n}=12, \mathrm{p}=0.119)$. After performing two tailed $t$ test for paired data, there was no significant difference between paternal and maternal transmission for motor symptoms. Data were not available for psychiatric onset in parents.

\section{ApoE and age of onset}

No statistically relevant difference was detectable either in motor or in psychiatric age of onset between the different ApoE genotypes (table 2). Combining all patients with $\in 4$ alleles into one group, the mean (SD) age of onset of motor and psychiatric symptoms was $45.71(9.29)$ years $(n=41)$ and 44.69 (8.21) years $(\mathrm{n}=32)$, respectively. In six patients it was not possible to determine a genotype for ApoE due to technical reasons. In order to exclude possible interference by the number of CAG repeats in our analysis, we performed multivariate regression analysis using the age of onset as a dependent variable and ApoE genotype and CAG repeat length as independent variables. There was no independent correlation for the ApoE genotypes and age of onset of first motor symptoms in any comparison $(\epsilon 2 \epsilon 3 v \in 3 \in 3: \mathrm{p}=0.623$; $\epsilon 3 \in 3 v \in 3 \in 4: \mathrm{p}=0553 ; \epsilon 3 \in 3 v$ combination of $\epsilon 3 \in 4$ and $\epsilon 4 \in 4$ : $\mathrm{p}=0.458$ ). No regression analysis with the subgroup $\in 4 \in 4$ was performed because of the low number of patients $(n=3)$. Also, there was no independent correlation for the ApoE genotypes and age of onset of psychiatric symptoms in any comparison. After fitting an exponential model for the dependence between CAG repeat number and age of onset, we could not identify significant differences in age of onset for motor and psychiatric symptoms. Distribution of the ApoE alleles was 0.0759 for $\epsilon 2,0.7724$ for $\epsilon 3$, and 0.1517 for $\epsilon 4$.

\section{ApoE genotypes and sex}

There was no independent correlation for the ApoE genotypes and age of onset of motor signs in any comparison for male $(\in 2 \in 3 \quad v \in 3 \in 3: \mathrm{p}=0.543 ; \epsilon 3 \in 3 \quad v \in 3 \in 4: \mathrm{p}=0.222 ; \epsilon 3 \in 3 \quad v$ combination of $\epsilon 3 \in 4$ and $\epsilon 4 € 4: \mathrm{p}=0.318$ ) or female patients $(\epsilon 2 € 3 \quad v \quad \epsilon 3 \epsilon 3: \mathrm{p}=0.855 ; \epsilon 3 \epsilon 3 \quad v \in 3 \in 4: \mathrm{p}=0.745 ; \epsilon 3 \epsilon 3 \quad v$ combination of $\epsilon 3 \in 4$ and $\epsilon 4 \epsilon 4: \mathrm{p}=0.949)$. Also, no significant difference in age of onset for first psychiatric abnormalities was found.

Significant differences between sexes were absent in age of onset of motor and psychiatric symptoms within the different ApoE genotypes after excluding the influence of CAG repeat lengths (analysis of variance, CAG repeat length

Table 2 Age of onset of symptoms (motor/psychiatric) in patients according to ApoE genotype

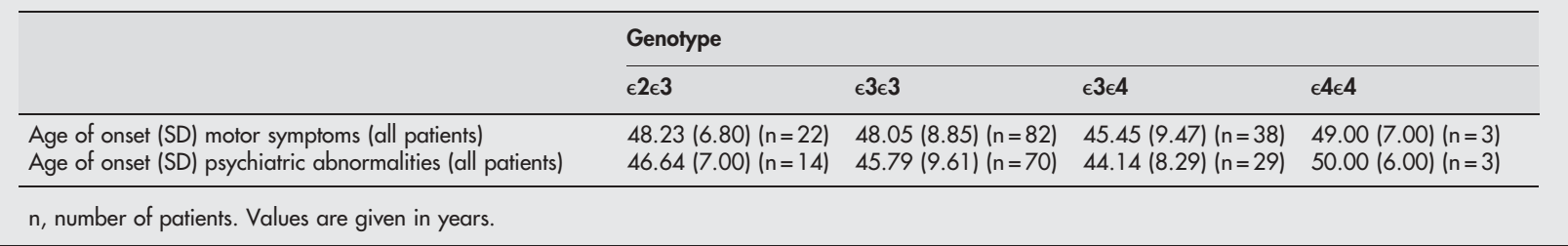


Table 3 Age of onset of motor/psychiatric symptoms according to ApoE genotype and sex

\begin{tabular}{|c|c|c|c|c|}
\hline & \multicolumn{4}{|l|}{ Genotype } \\
\hline & $\epsilon 2 \in 3$ & $\epsilon 3 \in 3$ & $\epsilon 3 \in 4$ & $\epsilon 4 € 4$ \\
\hline Age of onset (SD), motor symptoms (male patients) & $48.54(6.06)(n=13)$ & $47.45(7.97)(n=44)$ & $44.17(9.38)(n=18)$ & $57.00(n=1)$ \\
\hline Age of onset (SD), psychiatric symptoms (male patients) & $48.00(5.81)(n=9)$ & $45.10(9.18)(n=40)$ & $44.00(10.27)(n=14)$ & $56.00(n=1)$ \\
\hline Age of onset (SD), motor symptoms (female patients) & $47.78(8.12)(n=9)$ & $48.74(9.83)(n=38)$ & $46.60(9.64)(n=20)$ & $45.00(1.41)(n=2)$ \\
\hline Age of onset (SD), psychiatric symptoms (female patients) & $44.20(8.96)(n=5)$ & $46.70(10.25)(n=30)$ & $44.27(6.30)(n=15)$ & $47.00(4.24)(n=2)$ \\
\hline
\end{tabular}

as covariate). In particular, there was no significant sex difference in age of onset for first motor symptoms $(p=0.815)$ and first psychiatric abnormalities $(p=0.426)$ within the ApoE genotypes $€ 2 € 3$ after excluding the influence of CAG repeat length (table $3 ; t$ test).

\section{ApoE genotypes and clinical symptoms}

Differentiating the main psychiatric symptoms (dementia and depression) according to ApoE genotypes, there was no statistically relevant difference $\left(\chi^{2}: 2 \times 2\right.$ table, separate test for each ApoE genotype for example' $\epsilon 3 \in 4^{\prime} v$ not $\epsilon 3 \in 4^{\prime}$, $\mathrm{p}$ values between 0.12 and 0.70 before Bonferroni adjustment).

Concerning dementia as the main symptom we found 19 patients $(23.2 \%)$ with $\epsilon 3 \in 3$, but only four patients $(10.5 \%)$ with the $\in 3 \in 4$ genotype (table 4 ). This difference is not significant. Combining all genotypes containing the $\epsilon 4$ allele, we diagnosed dementia in $9.8 \%$, psychosis in $7.3 \%$, depression in $26.8 \%$, choreic movements in $53.6 \%$, and other movement disturbances in $2.4 \%$. These results, however, are not significant.

\section{CONCLUSION}

In HD the age of onset is known to be negatively correlated with the lengths of the expanded CAG blocks in the huntingtin gene. ${ }^{3}$ This variation in CAG block length may explain some of the variation in age of onset. Yet, particularly in those cases with lower CAG repeat numbers, there is a high variance in age of onset. Therefore, other genetic variables have been assumed. In order to investigate these genetic influences, we assembled a homogenous cohort of patients harbouring expansions of between 41 and 45 CAG repeats. Remarkably, we found a significantly negative correlation even in this narrow range of repeat lengths between block length and age of onset for motor symptoms as well as for psychiatric abnormalities. This correlation persisted even after subdividing this cohort by sex.

In our cohort, first psychiatric abnormalities appeared approximately 1.3 years earlier than motor symptoms, thus confirming the findings of other authors. ${ }^{18}{ }^{19}$ However, in general the diagnosis of first psychiatric signs due to HD appears less certain than the diagnosis of motor onset because of the occurrence of unspecific or reactive abnormalities in patients and persons at risk from HD families. The diagnosis of psychiatric onset, therefore, might be less important for the investigation of parameters of influence in the disease.
The $\epsilon 4$ allele of the ApoE genotype has been reported to delay the age of onset of patients with $\mathrm{HD}^{13}$ and to exert gender specific effects upon an earlier age of onset due to the $\epsilon 2 \epsilon 3$ genotype in males with HD. ${ }^{14}$ Effects of the ApoE genotypes were not described in other studies. ${ }^{511}$ In the former investigation information is lacking about whether patients were related. The studies revealing delayed age of onset in HD were designed to rule out the influence of genetic variables other than CAG repeats. A broad range of CAG length had been investigated in smaller cohorts. In order to diminish the influence of CAG repeat length we studied the effect of the ApoE genotypes in 145 patients. Comparing the data of our cohort to data of healthy controls from the literature there was no relevant difference in distribution of the ApoE alleles. In particular to exclude a bias the frequency of the $\epsilon 4$ allele was distributed equally compared to normal controls. ${ }^{20}$ By using the same statistical methods as described in the abovementioned reports, we cannot confirm any relevant effect of the $\epsilon 4$ allele or of the $\epsilon 2 \epsilon 3$ genotype. Moreover, we did not find any gender specific effect for ApoE after stratifying our cohort by sex. On the contrary, the mean age of onset in our cohort was even slightly earlier in patients with $€ 4$ genotypes as compared to $€ 3 € 3$, both for motor as well as for psychiatric onset. The negative effects of the $\in 4$ allele concerning age of onset have also been described in other neurodegenerative diseases, especially AD. Therefore, our data are more consistent with the common concept of the influence of ApoE on neurodegeneration.

Possible explanations for the differences between our findings and those of the other groups are: (1) other authors used a linear model for statistical analysis. We presume that, especially in higher CAG length ranges, the use of exponential statistical models is more appropriate. This assumption is supported by recent results, that is, that neurodegeneration follows exponential rather than linear decline in animal models $^{21}$ and in man.22 Our results did not reveal any statistical significance either in linear or in exponential models; (2) longer CAG blocks induce different pathomechanism(s) and cellular interaction patterns with ApoE than the lower ranges. Such an interpretation, however, would fail to explain the findings in the larger study ${ }^{5}$ involving a broad range of CAG lengths and using logarithmic transformation, without revealing any effect of ApoE genotypes on age of onset in HD.

There was no significant clinical difference the regarding main psychiatric symptoms of the patients after genotyping

Table 4 Main symptoms of patients according to ApoE genotype

\begin{tabular}{cclcll}
\hline $\begin{array}{l}\text { ApoE } \\
\text { allele }\end{array}$ & $\begin{array}{l}\text { Dementia, } \\
\mathbf{n}(\%)\end{array}$ & $\begin{array}{l}\text { Psychosis, } \\
\mathbf{n}(\%)\end{array}$ & $\begin{array}{l}\text { Depression, } \\
\mathbf{n}(\%)\end{array}$ & $\begin{array}{l}\text { Choreic } \\
\text { movements, }\end{array}$ & $\begin{array}{l}\text { O (\%) } \\
\text { disturbances, } \mathbf{n}(\%)\end{array}$ \\
\hline 0203 & $5(22.7 \%)$ & $2(9.1 \%)$ & $4(18.2 \%)$ & $11(50.0 \%)$ & $0(0.0 \%)$ \\
0303 & $19(23.2 \%)$ & $5(6.1 \%)$ & $16(19.5 \%)$ & $39(47.5 \%)$ & $3(3.7 \%)$ \\
0304 & $4(10.5 \%)$ & $3(7.9 \%)$ & $9(23.7 \%)$ & $21(55.3 \%)$ & $1(2.6 \%)$ \\
0404 & $0(0.0 \%)$ & $0(0.0 \%)$ & $2(66.7 \%)$ & $1(33.3 \%)$ & $0(0.0 \%)$ \\
\hline
\end{tabular}


for ApoE. In our cohort, patients with dementia as the main symptom appear less frequently in the $\epsilon 4$ group than in the other subgroups. Although this finding is not significant, different pathomechanisms may be relevant in HD and in $\mathrm{AD}$. We cannot assume that ApoE genotypes have a similar influence on the development of dementia as in AD. Thus we conclude, that ApoE is not a modifying factor for age of onset in HD.

\section{Authors' affiliations}

C Saft, J E Andrich, N Brune, P H Kraus, H Przuntek, Huntington Center NRW, Department of Neurology, St Josef Hospital, Ruhr-University Bochum, Germany

M Gencik, J T Epplen, Huntington Center NRW, Department of Human Genetics, Ruhr-University Bochum, Germany

This study was supported by FORUM, Medical Faculty, Ruhr-University Bochum (F269/01)

Competing interests: none declared

\section{REFERENCES}

1 The Huntington's Disease Collaborative Research Group. A novel gene containing a trinucleotide repeat that is expanded and unstable on Huntington's disease chromosomes. Cell 1993;72:971-83.

2 Feigin A, Zgaljardic D. Recent advances in Huntington's disease: implications for experimental therapeutics. Curr Opin Neurol 2002;15:483-9.

3 Snell RG, MacMillan JC, Cheadle JP, et al. Relationship between trinucleotide repeat expansion and phenotypic variation in Huntington's disease. Nat Genet 1993;4:393-7.

4 Andrew SE, Goldberg YP, Kremer B, et al. The relationship between trinucleotide (CAG) repeat length and clinical features of Huntington's disease. Nat Genet 1993;4:398-403.

5 Rubinsztein DC, Leggo J, Chiano M, et al. Genotypes at the GluR6 kainate receptor locus are associated with variation in the age of onset of Huntington disease. Proc Natl Acad Sci U S A 1997;94:3872-6.

6 Rubinsztein DC, Leggo J, Coles R, et al. Phenotypic characterization of individuals with $30-40$ CAG repeats in the Huntington disease (HD) gene reveals HD cases with 36 repeats and apparently normal elderly individuals with 36-39 repeats. Am J Hum Genet 1996;59:16-22.

7 Mahley RW, Rall SC Jr. Apolipoprotein E: far more than a lipid transport protein. Annu Rev Genomics Hum Genet 2000;1:507-37.

8 Mahley RW, Huang Y. Apolipoprotein E: from atherosclerosis to Alzheimer's disease and beyond. Curr Opin Lipidol 1999;10:207-17.

9 Strittmatter WJ, Saunders AM, Schmechel D, et al. Apolipoprotein E: highavidity binding to beta-amyloid and increased frequency of type 4 allele in late-onset familial Alzheimer disease. Proc Natl Acad Sci U S A 1993;90:1977-81.

10 Corder EH, Saunders AM, Strittmatter WJ, et al. Gene dose of apolipoprotein E type 4 allele and the risk of Alzheimer's disease in late onset families. Science 1993;261:921-3.

11 Kalman J, Juhasz A, Majtenyi K, et al. Apolipoprotein E polymorphism in Pick's disease and in Huntington's disease. Neurobiol Aging 2000;21:555-8.

12 Krüger R, Vieira-Saecker AM, Kuhn W, et al. Increased susceptibility to sporadic Parkinson's disease by a certain combined alpha-synuclein/ apolipoprotein E genotype. Ann Neurol 1999;45:611-7.

13 Panas M, Avramopoulos D, Karadima G, et al. Apolipoprotein E and presenilin-1 genotypes in Huntington's disease. J Neurol 1999;246:574-7.

14 Kehoe P, Krawczak M, Harper PS, et al. Age of onset in Huntington disease: sex specific influence of apolipoprotein $E$ genotype and normal $C A G$ repeat length. J Med Genet 1999;36:108-1 11

15 Saft C, Andrich J, Meisel NM, et al. Assessment of complex movements reflects dysfunction in Huntington's disease. J Neurol 2003;250(12):1469-74.

16 Warner JP, Barron LH, Brock DJ. A new polymerase chain reaction (PCR) assay for the trinucleotide repeat that is unstable and expanded on Huntington's disease chromosomes. Mol Cell Probes 1993;7:235-9.

17 Riess O, Noerremoelle A, Soerensen SA, et al. Improved PCR conditions for the stretch of (CAG)n repeats causing Huntington's disease. Hum Mol Genet 1993;2:637.

18 Leroi I, Michalon M. Treatment of the psychiatric manifestations of Huntington's disease: a review of the literature. Can J Psychiatry 1998;43:933-40

19 Paulsen JS, Zhao H, Stout JC, et al. Clinical markers of early disease in persons near onset of Huntington's disease. Neurology 2001 ;57:658-62.

20 Corbo RM, Scacchi R. Apolipoprotein E (APOE) allele distribution in the world. Is APOE*4 a 'thritty' allele? Ann Hum Genet 1999;63(pt 4): 301-10.

21 Clarke G, Collins RA, Leavitt BR, et al. A one-hit model of cell death in inherited neuronal degenerations. Nature 2000;406:195-9.

22 Perutz MF, Windle AH. Cause of neural death in neurodegenerative diseases attributable to expansion of glutamine repeats. Nature $2001 ; 412$ :143-4. 